\title{
Prevalência de bacilos Gram-negativos não fermentadores de pacientes internados em Porto Alegre-RS
}

\section{Prevalence of non-fermenting Gram-negative bacilli among inpatients from Porto Alegre-RS}

Bruno Deliberali'; Kendi Nishino Myiamoto²; Carlos Hugo Del Priore Winckler Neto ${ }^{3}$; Rafael Silvio Remus Pulcinelli4; Alzira Resende do Carmo Aquino ${ }^{5}$; Bruno Stefanello Vizzotto ${ }^{6}$; Roberto Christ Vianna Santos 7

unitermos

Bacilos Gram-negativos não fermentadores

Pseudomonas aeruginosa Antimicrobianos

\section{resumo}

Introdução: Os bacilos Gram-negativos não fermentadores (BGNNF) são frequentemente associados às infecções hospitalares. Além da alta incidência, esses microrganismos possuem resistência a diversos antimicrobianos. Objetivo: Analisar a prevalência e o perfil de resistência de BGNNF. Métodos: Foram analisados 14.971 laudos de pacientes em um hospital privado de Porto Alegre-RS, no período de maio de 2006 a março de 2008, sem distinção de sexo e idade. Resultados e conclusão: Foram isoladas 326 amostras de BGNNF. As espécies mais prevalentes foram: Pseudomonas aeruginosa (65,03\%), Acinetobacter baumannii (16,56\%) e Stenotrophomonas maltophilia (9,5\%). Outras espécies apresentaram índices inferiores a 5\%. Os microrganismos foram isolados de diversos sítios infecciosos. Os materiais biológicos que apresentaram maior positividade para esses microrganismos foram o aspirado traqueal (38,34\%), o escarro (18,71\%) e a urina (15,95\%). A resistência bacteriana mostrou-se mais expressiva a tetraciclinas $(89,57 \%)$ e sulfametoxazol/trimetoprima $(79,75 \%)$. Os antimicrobianos mais ativos foram polimixina B, com $100 \%$ de sensibilidade, e piperaciclina/tazobactam, com $75,2 \%$ de sensibilidade.

\section{abstract}

Introduction: The non-fermenting Gram-negative bacilli (NFGNB) have been widely associated with nosocomial infections. Not only are these microorganisms highly prevalent but they are also highly resistant to NFGNB. Objective: To assess the prevalence and resistance profile of non-fermenting Gram-negative bacilli. Methods: 14.971 patient reports from a private hospital in Porto Alegre, Rio Grande do Sul, from May/2006 to March/2008 were analyzed. Results and conclusion: Three hundred twenty-six samples of non-fermenting Gram-negative bacilli were isolated. The most prevalent species were Pseudomonas aeruginosa (65.03\%), Acinetobacter baumannii (16.56\%), and Stenotrophomonas maltophilia (9.5\%). Other species showed rates lower than 5\%. The microorganisms were isolated from several infectious sites and the biological materials that showed higher positivity were the following: tracheal aspirate (38.34\%), spittle (18.71\%) and urine (15.95\%). Bacterial resistance was higher with tetracyclines (89.57\%) and sulfamethoxazole/trimethoprim (79.75\%). The most active antimicrobials were polymyxin B and piperacillin/tazobactam with $100 \%$ and $75.2 \%$ sensibility, respectively. key words

Non-fermenting Gram-negative bacilli

Pseudomonas aeruginosa

Antimicrobials

\footnotetext{
1. Especialista em Análises Clínicas; farmacêutico bioquímico.

2. Mestre em Biologia Celular e Molecular; biomédico.

3. Mestre em Ciências Farmacêuticas; farmacêutico bioquímico.

4. Especialista em Microbiologia Clínica; farmacêutico bioquímico.

5. Mestra em Biologia Celular e Molecular; farmacêutica bioquímica.

6. Mestre em Ciências Farmacêuticas; professor auxiliar do Centro Universitário Franciscano (UNIFRA).

7. Doutor em Biologia Celular e Molecular; professor adjunto do UNIFRA.
} 


\section{Introdução}

Os bacilos Gram-negativos não fermentadores (BGNNF) constituem um grupo extremamente diverso ${ }^{(23)}$, são estritamente aeróbios, não esporulados e se caracterizam pelo fato de serem incapazes de utilizar carboidratos como fonte de energia por meio de fermentação, degradando-os pela via oxidativa ${ }^{(1,23,26)}$.

As infecções por BGNNF aumentaram no grau de importância em instituições hospitalares a partir da década de 1970, tendo como principais representantes Pseudomonas aeruginosa, Acinetobacter spp., Stenotrophomonas maltophilia e Burkholderia cepacia ${ }^{(3,11,5)}$. Além disso, esses não fermentadores vêm apresentando sensibilidade diminuída a um grande número de fármacos ${ }^{(34)}$. Essas infecções têm origem endógena ou exógena, dependendo de diversos fatores, como uso de substâncias imunossupressoras, utilização abusiva de agentes antimicrobianos de amplo espectro, procedimentos cirúrgicos prolongados e instrumentação mecânica inadequada ${ }^{(8,10,12)}$. Esses microrganismos estão relacionados predominantemente com infecções hospitalares ${ }^{(14,28)}$.

O objetivo principal deste trabalho foi verificar a prevalência de isolamento e o perfil de resistência dos BGNNF em pacientes hospitalizados em um hospital privado de Porto Alegre-RS, no período de maio de 2006 a março de 2008.

\section{Materiais e métodos}

Realizou-se uma análise retrospectiva dos prontuários de registro do Laboratório de Microbiologia Clínica do Hospital Divina Providência de Porto Alegre no período de maio de 2006 a março de 2008. Foram utilizados laudos de pacientes hospitalizados sem distinção de sexo e idade. O presente trabalho foi aprovado pelo Comitê de Ética em Pesquisa com Seres Humanos do Centro Universitário Franciscano (UNIFRA).

\section{Resultados e discussão}

Neste estudo foram analisados 14.971 laudos de pacientes e, embora não tenhamos feito distinção das unidades hospitalares em que esses pacientes se encontravam, pudemos verificar que os microrganismos citados anteriormente são importantes agentes causadores de infecções hospitalares, sendo patógenos oportunistas, como encontrado na literatura ${ }^{(20,26,35)}$, e isso ocorre devido a alguns fatores como, por exemplo, a queda da imunidade dos pacientes e a utilização de procedimentos invasivos.

A frequência geral de BGNNF em nosso estudo foi de 326 (2,18\%), conforme mostrado na Tabela 1. Embora esses dados sejam comparados com o número global de amostras analisadas e não somente com as culturas positivas, eles se aproximam do encontrado na literatura. Em estudo semelhante, Menezes et al. ${ }^{(26)}$ demonstraram que a frequência para esses microrganismos foi de $9,4 \%$.

\section{BGNNF isolados de pacientes} hospitalizados no Hospital Divina

Tabela 1 Providência de Porto Alegre-RS

\begin{tabular}{lcc}
\hline Microrganismo & $n$ & $\%$ \\
\hline Pseudomonas aeruginosa & 212 & 65 \\
\hline Acinetobacter baumannii & 54 & 16,5 \\
\hline Stenotrophomonas maltophilia & 31 & 9,5 \\
\hline Pseudomonas sp. & 12 & 3,7 \\
Acinetobacter sp. & 11 & 3,4 \\
\hline Alcaligenes sp. & 3 & 0,9 \\
\hline Sphingomonas paucimobilis & 2 & 0,6 \\
\hline Burkholderia cepacia & 1 & 0,3 \\
\hline Total de não fermentadores & 326 & 100 \\
\hline
\end{tabular}

BGNNF: bacilos Gram-negativos não fermentadores.

A frequência de Pseudomonas aeruginosa apresentada em nosso estudo foi similar à relatada por Mimica et al.(27) e Frota et al. ${ }^{(17)}$ e superior à encontrada por Menezes et al. ${ }^{(26)}$. Menezes et al. ${ }^{(25)}$, estudando culturas de ponta de cateter, também apontaram esse microrganismo como o mais comumente isolado entre os BGNNF. Além disso, vários pesquisadores têm estudado essa bactéria devido a sua alta frequência de isolamento, o que vem ao encontro do relatado em nosso trabalho ${ }^{(5,14)}$.

O estudo também mostrou grande similaridade com o relatado por Koneman et al. ${ }^{(23)}$, cuja prevalência de Pseudomonas aeruginosa foi de $66 \%$ e de Acinetobacter baumannii, 7\%, e que está em acordo com o descrito por Karloswky et al. ${ }^{(22)}$ e Shah et al.(36), que destacaram esses patógenos como os não fermentadores mais isolados em espécimes clínicos hospitalares.

É importante ressaltar neste artigo a diversidade de sítios infecciosos, ou seja, o tipo de material biológico no qual foram isolados esses microrganismos. A maioria dos BGNNF analisados foram isolados de aspirado traqueal $(125 ; 38,3 \%)$, escarro $(61 ; 18,7 \%)$ e urina $(52 ; 15,9 \%)$. 
Ainda encontramos cepas em lavado broncoalveolar (30; $9,2 \%)$, sangue $(19 ; 5,8 \%)$, secreção de ferida operatória $(11 ; 3,4 \%)$, secreção abdominal $(6 ; 1,8 \%)$, ponta de cateter $(6 ; 1,8 \%)$ e outros (secreção vaginal, líquido pleural, água de diálise, líquido peritoneal, secreção do braço direito, espermatozoide, líquido pélvico etc.), como pode ser visualizado na Tabela 2.

\begin{tabular}{lcc} 
& $\begin{array}{l}\text { Espécimes clínicos de pacientes } \\
\text { hospitalizados do Hospital Divina }\end{array}$ \\
Tabela 2 & \begin{tabular}{l} 
Providência de Porto Alegre-RS \\
\hline Sítio infeccioso
\end{tabular} & $\%$ \\
\hline Aspirado traqueal & 125 & 38,3 \\
\hline Escarro & 61 & 18,7 \\
\hline Urina & 52 & 15,9 \\
\hline Lavado broncoalveolar & 30 & 9,2 \\
Sangue & 19 & 5,8 \\
\hline Secreção de ferida operatória & 11 & 3,4 \\
\hline Secreção abdominal & 6 & 1,8 \\
Ponta de cateter & 6 & 1,8 \\
\hline Outros & 16 & 4,9 \\
\hline Total & 326 & 100 \\
\hline
\end{tabular}

Com isso, pode-se perceber que a maioria das amostras foi isolada a partir do material do trato respiratório (68\%). Esse fato também foi constatado por Menezes et al. ${ }^{(26)}$, o que de certa forma já era esperado, uma vez que relatamos, em nosso estudo, grande quantidade de cepas isoladas de Pseudomonas aeruginosa, microrganismo comum em patologias respiratórias ${ }^{(13,15,35)}$. Estudando esse patógeno, encontramos a maioria de cepas provindas do trato respiratório.

Nas patologias respiratórias também é muito frequente o isolamento de outros não fermentadores. Garcia et al.(20), estudando a Stenotrophomonas maltophilia, encontraram $70 \%$ de cepas do trato respiratório para esse bacilo e Coronas et al. ${ }^{(6)}$, estudando indivíduos com infecção/colonização por Acinetobacter baumannii, relataram que $45 \%$ deles apresentavam enfermidade respiratória crônica. $O$ trato urinário vem logo em seguida com 52 isolados (15,9\%), sendo um pouco inferior ao encontrado por Menezes et al. ${ }^{(26)}$ e Figueiredo et al. ${ }^{(15)}$, este último, em relação a Pseudomonas aeruginosa.

Quando analisamos o perfil de resistência dos BGNNF, verificamos importantes índices de resistência diante de diversos antimicrobianos, como pode ser visto na Figura.

O antimicrobiano mais ativo foi a polimixina B com $100 \%$ de sensibilidade, embora tenha sido reportado

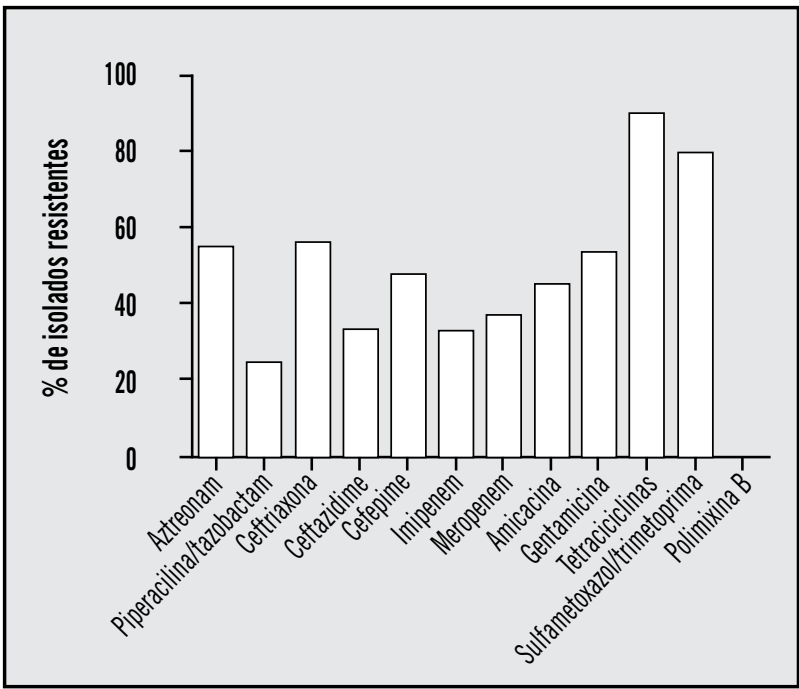

Figura - Perfil de resistência aos antimicrobianos de pacientes hospitalizados no Hospital Divina Providência em Porto Alegre-RS

apenas para amostras que apresentaram resistência a todos os outros antimicrobianos. Esse fato foi relatado em nosso país $(2,16,18,29,32,38)$ e é preocupante, visto que a perda de sensibilidade em curto espaço de tempo não corresponde à velocidade com que novos antimicrobianos podem ser lançados no mercado, deixando os pacientes expostos a infecções com poucas possibilidades de tratamento( ${ }^{(7)}$.

Outro antimicrobiano que apresentou grande atividade contra os não fermentadores foi a piperacilina associada ao tazobactam $(75,2 \%)$, relato semelhante ao encontrado pelos pesquisadores do programa SENTRY ${ }^{(35)}$, na América Latina, em relação a Pseudomonas aeruginosa, não se afastando também do que foi dito por Figueiredo et al. ${ }^{(15)}$ para essa mesma bactéria.

Gentamicina, ceftriaxona e aztreonam obtiveram resultados de resistência intermediária, com 53,4\% para a primeira e $55,2 \%$ para as últimas. Quase $50 \%$ dos isolados não fermentadores foram resistentes à cefepima, uma cefalosporina de quarta geração, justificando a preocupação mundial com relação à resistência a esses microrganismos.

Os carbapenêmicos são conhecidos como potentes agentes para o tratamento de infecções por Pseudomonas aeruginosa e Acinetobacter spp. ${ }^{(24,31,33,37)}$, bactérias com maior prevalência em nosso estudo. Isso foi comprovado, já que encontramos apenas $32,8 \%$ e $37,1 \%$ de isolados resistentes a imipenem e meropenem, respectivamente. Porém, nosso trabalho sugere aumento da resistência a esses fármacos, uma vez que no estudo com enfoque em imipenem, Freitas et al. ${ }^{(16)}$ ressaltaram resistência de $16,3 \%$ pela Pseudomonas aeruginosa, índice semelhante ao encontrado pelo programa SENTRY no Brasil e na América Latina ${ }^{(35)}$. 
Em outro estudo recente, Bier et al. ${ }^{(4)}$ detectaram resistência a carbapenêmicos mediada por carbapenemases da classe D (OXA-23) em isolados de Acinetobacter baumannii em Curitiba-PR.

Comparando a atividade in vitro desses carbapenêmicos, vários autores demonstraram que o meropenem possui atividade contra Gram-negativos superior à do imipenem $^{(9,19,30)}$, fato que não foi confirmado na nossa pesquisa, visto que encontramos maior número de amostras resistentes a meropenem.

Por outro lado, os maiores níveis de resistência se expressaram a tetraciclinas $(89,6 \%)$ e sulfametoxazol/trimetoprima $(79,7 \%)$, fato também relatado por Menezes et al. (26) e que pode estar relacionado com o acesso facilitado a esses medicamentos, já que são fármacos baratos e de fácil aquisição, e o uso indiscriminado pode, por pressão, selecionar cepas resistentes. Hinrichsen ${ }^{(21)}$ descreve que 0 uso frequente de determinado antimicrobiano é capaz de elevar proporcionalmente a resistência bacteriana a esse fármaco, e o hospital que faz uso de grandes quantidades de um antimicrobiano acabará apresentando, em um futuro próximo, problemas associados à resistência a esse fármaco.

\section{Conclusão}

Dos 14.971 laudos analisados, em 326 (2,18\%) foram identificados BGNNF. Apesar da baixa prevalência com que aparecem na rotina, os BGNNF têm significativa importância pelo risco que proporcionam aos pacientes. Esses microrganismos podem expressar vários fatores de virulência como forma de defesa contra o hospedeiro, facilitando sua invasão e sua disseminação no organismo humano. Além disso, apresentam altas taxas de morbimortalidade e resistência aos antimicrobianos, restringindo a escolha terapêutica.

De acordo com a Tabela 1, a espécie bacteriana Pseudomonas aeruginosa foi o microrganismo com maior frequência, como já era esperado, o que demonstra ser um importante patógeno nosocomial. Entretanto, um dos resultados mais interessantes em nosso estudo foi a alta prevalência do gênero Acinetobacter, confirmando ser um microrganismo emergente no meio hospitalar.

Mediante a Tabela 2, podemos analisar que a maioria dos isolados em nossa pesquisa foi proveniente de material do trato respiratório. Isso se deve à grande prevalência de Pseudomonas aeruginosa e Acinetobacter sp., não fermentadores conhecidos por provocar infecções respiratórias.

Os antimicrobianos mais eficazes contra essas bactérias foram a polimixina $B$ e a piperacilina associada ao tazobactam, representando ser boa alternativa terapêutica no tratamento dessas infecções. Também podemos concluir que os carbapenêmicos continuam sendo escolhas adequadas no tratamento dessas infecções, embora se tenha notado um acréscimo na resistência a esses antimicrobianos.

Os menos eficazes foram as tetraciclinas e o sulfametoxazol associado à trimetoprima, o que de algum modo é explicado pelo uso indiscriminado desses fármacos empírica e profilaticamente.

Nossos resultados destacam a importância de trabalhos como este para guiar a terapia e as medidas de controle de infecção, uma vez que existe variação dos padrões de resistência de país para país e mesmo entre diferentes hospitais em uma cidade. Assim, a criação de um sistema de monitoramento da resistência ajudaria muito na escolha da terapia empírica mais adequada.

Para que haja diminuição desses números de resistência no país e no mundo é preciso ainda que ocorra a implementação de sistemas de vigilância epidemiológica e de medidas de controle de infecção. Dessa maneira, esperamos que o nosso trabalho auxilie na adoção de políticas concretas com relação à utilização racional de antimicrobianos e no controle intra-hospitalar de infecções, reduzindo o aparecimento de microrganismos multirresistentes.

\section{Referências}

1. ANVISA. Agência Nacional de Vigilância Sanitária. Detecção e identificação de bactérias de importância médica, 2004. Disponível em: <http://www.anvisa.gov.br/ servicosaude/manuais/microbiologia/mod_5_2004. pdf>. Acesso em: 23 set. 2008.
2. ARRUDA, E. A. et al. Nosocomial infections caused by multiresistant Pseudomonas aeruginosa. Infect Control Hosp Epidemiol, n. 20, p. 620-3, 1999.

3. BERGOGNE-BÉRÉZIN, E.; TOWNER, K. J. Acinetobacter spp. as nosocomial pathogens: microbiological, clinical, 
and epidemiological features. Clin Microbiol Rev, n. 9, p. 148-65, 1996.

4. BIER, K. E. S. et al. Temporal evolution of carbapenem resistant Acinetobacter baumannii in Curitiba, southern Brazil. Am J Infect Control, n. 38, p. 308-14, 2010.

5. CEZÁRIO, R. C. et al. Nosocomial outbreak b yimipenemresistant metallo-b-lactamase-producing Pseudomonas aeruginosa in an adult intensive care unit in a Brazilian teaching hospital. Enferm Infecc Microbiol Clin, v. 27, n. 5, p. 269-74, 2009.

6. CORONAS, S. J. et al. Infección/colonización nosocomial de las vías respiratorias por Acinetobacter baumannii en una planta de medicina interna. Anales de Medicina Interna, v. 19, n. 10, p. 511-4, 2002.

7. DAGATA, E. M. Rapidly rising prevalence of nosocomial multidrug-resistant, Gram-negative bacilli: a 9-year surveillance study. Infect Control Hosp Epidemiol, v. 10, n. 25, p. 842-6, 2004.

8. DANCE, D. A. B. Meliodosis. Culture, n. 15, p. 2-4, 1994.

9. EDWARDS, J. R.; BETTS, M. J. Carbapenems: the pinnacle of the B-lactam antibiotics or room for improvement. J Antimicrob Chemother, n. 45, p. 1-4, 2000.

10. FADEN, H.; HARABUCHI, Y.; HONG, J. J. Epidemiology of Moraxella catarrhalis in children during the first 2 years of life: relationship to otitis media. J Infect Dis, n. 169, p. 1312-7, 1994.

11. FALAGAS, M. E.; KOPTERIDES, P. Risk factors for the isolation of multi-drug-resistant Acinetobacter baumannii and Pseudomonas aeruginosa:a systematic review of the literature. J Hosp Infect, n. 64, p. 7-15, 2006.

12. FERGIE, J. E. et al. Pseudomonas aeruginosa bacteremia in immunocompromised children: analysis of factors associated with a poor outcome. Clin Infect Dis, n. 18, p. 390-4, 1994.

13. FERRAREZE, M. V. G. et al. Pseudomonas aeruginosa multiresistente em unidade de cuidados intensivos: desafios que procedem? Acta Paul Enferm, v. 1, n. 20, p. 7-11, 2007.

14. FIGUEIREDO-MENDES, C. M. et al. Pseudomonas aeruginosa clonal dissemination in Brazilian intensive care units. Enferm Infecc Microbiol Clin, v. 23, n. 7, p. 402-5, 2005.

15. FIGUEIREDO, P. A. E. et al. Pseudomonas aeruginosa: freqüência de resistência a múltiplos fármacos e resistência cruzada entre antimicrobianos no Recife/ PE. Revista Brasileira de Terapia Intensiva, v. 4, n. 19, p. 421-7, 2007.

16. FREITAS, A. L.; BARTH, A. L. Antibiotic resistance and molecular typing of Pseudomonas aeruginosa: focus on imipenem. Braz J Infect Dis, v. 1, n. 6, p. 1-7, 2002.

17. FROTA, C. C.; MOREIRA, J. L. B. Frequency of nonfermentative Gram-negative bacilli isolated from clinical material of patients at Universidade Federal do Ceará Hospital complex Brazil. Rev Microbiol, v. 29, n. 3, p. 25-31, 1998.

18. GALES, A. C. et al. Carbapenem-resistant Pseudomonas aeruginosa outbreak in an intensive care unit of a teaching hospital. The Brazilian Journal of Infectious Diseases, v. 4, n. 8, p. 267-71, 2004.

19. GALES, A. C. et al. Comparative in vitro activity of meropenem versus other extended-spectrum antimicrobial agents against 2,085 clinical isolates tested in 13 Brazilian centers. Braz J Infect Dis, n. 1, p. 294-305, 1997.

20. GARCIA, O. D. et al. Proteases (caseinase and elastase), hemolysins, adhesion and susceptibility to antimicrobials of Stenotrophomonas maltophilia isolates obtained from clinical specimens. Brazilian Journal of Microbiology, n. 33, p. 157-62, 2002.

21. HINRICHSEN, S. L. Biossegurança e controle de infecções - risco sanitário hospitalar. Rio de Janeiro: Medsi, 2004.

22. KARLOWSKY, J. A.; DRAGHI, D. C.; JONES, M. E. Surveillance for antimicrobial susceptibility among clinical isolates of Pseudomonas aeruginosa and Acinetobacter baumannii from hospitalized patients in the United States, 1998 to 2001. Antim Ag Chemother, n. 47, p. 1681-8, 2003.

23. KONEMAN, E. W. et al. Diagnóstico microbiológico: texto e atlas colorido. 5. ed. Rio de Janeiro: Medsi, 2001.

24. LIVERMORE, D. M. Multiple mechanisms of antimicrobial resistance in Pseudomonas aeruginosa: our worst nightmare? Clin Infect Dis, n. 34, p. 634-40, 2002.

25. MENEZES, E. A. et al. Caracterização bacteriana em culturas de ponta de cateter. RBAC, v. 3, n. 34, p. 151-4, 2002.

26. MENEZES, E. A. et al. Perfil de infecção e resistência aos antimicrobianos de bacilos Gram-negativos não fermentadores isolados no Laboratório de Patologia Clínica Dr. Edilson Gurgel, Santa Casa de Misericórdia de Fortaleza-CE. RBAC, v. 36, n. 4, p. 209-12, 2004.

27. MIMICA, I.; MIMICA, L. N. J. Aztreonam, atividade in vitro frente a bactérias Gram-negativas. Arq Bras Med, n. 65, p. 603-4, 1991.

28. NOUÉR, S. A. Aspectos clínicos e fatores de risco relacionados com colonização ou infecção por Pseudomonas aeruginosa multirresistente. 2007. $144 \mathrm{f}$. Tese (Doutorado em Medicina) - Programa de PósGraduação em Medicina, Universidade Federal do Rio de Janeiro, Rio de Janeiro, 2007.

29. PELLEGRINO, F. L. et al. Occurrence of a multidrugresistant Pseudomonas aeruginosa clone in different hospitals in Rio de Janeiro, Brazil. J Clin Microbiol, v. 7, n. 40, p. 2420-4, 2002.

30. PFALLER, M. A.; JONES, R. N. A review of the in vitro activity of meropenem and comparative antimicrobial agents tested against 30,254 aerobic and anaerobic pathogens isolated world wide. Diagn Microbiol Infect Dis, n. 28, p. 157-63, 1997.

31. POLLACK, M. Pseudomonas aeruginosa. In: MANDELL, G. L.; BENNETT, J. E.; DOLIN, R. Principles and practice of infectious diseases. 5. ed. New York: Chuchill Livingstone, 2000. p. 2310-35.

32. RIBEIRO, J. Caracterização microbiológica e epidemiológica de amostras clínicas de Pseudomona aeruginosa resistentes aos carbapenens isoladas no Hospital de Base do Distrito Federal, 2001-2002. 2004. Tese (Doutorado) - Universidade de Brasília, Brasília, 2004.

33. SADER, H. S. Antimicrobial resistance in Brazil: comparation of results from two multicenter studies. Braz J Infect Dis, n. 4, p. 91-9, 2000.

34. SADER, H. S. et al. Pathogen frequency and resistance patterns in Brazilian hospitals: summary of results from 
three years of the SENTRY Antimicrobial Surveillance Program. Braz J Infect Dis, n. 5, p. 200-14, 2001.

35. SADER, H. S. et al. Dissemination ans diversity of metallo$\beta$-lactamases in Latin America: report from the SENTRY antimicrobial surveillance program. International of Journal of Antimicrobiology Agents, v. 25, p. 57-61, 2004.

36. SHAH, S. S.; GALLAGHER, P. G. Complications of conjunctivitis caused by Pseudomonas aeruginosa in a newborn intensive care unit. Pediatr Infect Dis J, n. 17, p. 97-102, 1998.
37. TOGNIM, M. C. B. et al. Resistance trends of Acinetobacter $s p p$. in Latin America and characterization of international dissemination of mult-drug resistant strains: five-year report of the SENTRY Antimicrobial Surveillance Program. Int J Infect Dis, n. 8 p. 284-91, 2004.

38. ZAVASCKI, A. P.; CRUZ, R. P.; GOLDANI, L. Z. High rate of antimicrobial resistance in Pseudomonas aeruginosa at a tertiary-care teaching hospital in Southern Brazil. Infect Control Hosp Epidemiol, v. 10, n. 25, p. 805-7, 2004. 